The definitive version is available at www.blackwell-synergy.com (C) American Society for Photobiology 0031-8655/02 DOI: 10.1562/0031-8655(2002)0760373BEMBNA2.0.CO2 


\section{Bacteriochlorophyll $e$ Monomers, But Not Aggregates, Sensitize Singlet Oxygen: Implications For A Self-Photoprotection Mechanism In Chlorosomes}

Juan B. Arellano $^{* 1,2}$, Thor Bernt Melø ${ }^{1}$, Carles M. Borrego ${ }^{3}$ and K. Razi Naqvi ${ }^{1}$

${ }^{1}$ Department of Physics, Norwegian University of Science and Technology (NTNU), N-7491

Trondheim, Norway

${ }^{2}$ Institute of Natural Resources and Agrobiology (CSIC), Cordel de Merinas 52, E-37008

Salamanca, Spain and

${ }^{3}$ Institute of Aquatic Ecology, University of Girona, E-17071 Girona, Spain

*Corresponding author: Institute of Natural Resources and Agrobiology (CSIC), Cordel de Merinas 52, E-37008 Salamanca, Spain. Fax: +34 923 219609; e-mail: jarellano@gugu.usal.es

Key words: Aggregates, Bacteriochlorophyll $e$, Carotenoids, Chlorosomes, Photoprotection, Quantum yield, Singlet Oxygen, Triplet State

\footnotetext{
Abbreviations: BChl, bacteriochlorophyll; Car, carotenoid; Cfl, Chloroflexus; Chl, Chlorobium; $\Delta A(\lambda ; t)$, time-resolved, laser-induced changes in absorbance; $\mathrm{MB}$, methylene blue; MGDG, monogalactosyl diglyceride; $\mathrm{O}_{2}\left({ }^{1} \Delta_{\mathrm{g}}\right)$, singlet delta oxygen; $\mathrm{O}_{2}\left({ }^{3} \Sigma_{\mathrm{g}}{ }^{-}\right)$, ground state (triplet sigma) oxygen; $\Phi_{\Delta}$, quantum yield of singlet oxygen; TmS, Time-resolved triplet-minus-singlet; TX, Triton X-100.
} 


\section{ABSTRACT}

Sensitization of singlet oxygen, $\mathrm{O}_{2}\left({ }^{1} \Delta_{\mathrm{g}}\right)$, by bacteriochlorophyll $e$ (BChle) has been investigated to gain a better understanding of the photoprotection mechanism(s) operating in chlorosomes of green photosynthetic bacteria. The sensitization process has been studied in media where $\mathrm{BChle}$ forms monomers (acetone and aqueous solutions containing $0.5 \%$ Triton $\mathrm{X}-100(\mathrm{TX}))$, and in systems where BChle aggregates, namely aqueous solutions containing 0.003\% monogalactosyl diglyceride (MGDG) and chlorosomes (control as well as hexanolperturbed) from Chlorobium phaeobacteroides strain CL1401. In Ar-purged acetone, BChle triplets $\left(\mathrm{BChl} e^{\dagger}\right)$ have a lifetime of a few tens of $\mu$ s; however, in air-saturated acetone, quenching of BChl $e^{\dagger}$ by molecular oxygen, $\mathrm{O}_{2}\left({ }^{3} \Sigma_{\mathrm{g}}{ }^{-}\right)$, and formation of $\mathrm{O}_{2}\left({ }^{1} \Delta_{\mathrm{g}}\right)$ take place. The $\mathrm{O}_{2}\left({ }^{1} \Delta_{\mathrm{g}}\right)$ so formed is susceptible to quenching by BChle $e^{0}$, a ground-state BChle molecule. A Stern-Volmer analysis reveals a linear fit between the decay rate of $\mathrm{O}_{2}\left({ }^{1} \Delta_{\mathrm{g}}\right)$ and BChle concentration. The rate constants for the quenching of $\mathrm{O}_{2}\left({ }^{1} \Delta_{\mathrm{g}}\right)$ by $\mathrm{BChl} e^{0}$ and for the deactivation of $\mathrm{O}_{2}\left({ }^{1} \Delta_{\mathrm{g}}\right)$ by the solvent come out to be $k_{q}=(1.4 \pm 0.1) \times 10^{9} \mathrm{M}^{-1} \mathrm{~s}^{-1}$ and $k_{0}=(18.5 \pm 0.7) \times 10^{3} \mathrm{~s}^{-1}$, respectively. The absolute quantum yield of $\mathrm{O}_{2}\left({ }^{1} \Delta_{\mathrm{g}}\right)$ sensitization by BChle monomers is $0.65 \pm 0.15$ in air-saturated acetone. In aqueous phase, the triplet lifetime of BChle aggregates in native or hexanol-perturbed chlorosomes shortens by more than two orders of magnitude when compared to the triplet lifetime of BChle monomers in $0.5 \%$ TX solution (a few hundreds of $\mu \mathrm{s}$ ). Quenching by carotenoids makes only a minor contribution to the decay of BChle $e^{\dagger}$ in aggregates. Since $\mathrm{O}_{2}\left({ }^{1} \Delta_{\mathrm{g}}\right)$ sensitization by BChl $e^{\dagger}$ could be detected neither in MGDG aggregates nor in chlorosomes (control as well as hexanol-perturbed), it is concluded that $i$ ) this process is highly likely when BChle is present as a monomer, but not when it is tightly packed in artificial aggregates or in chlorosomes, and ii) carotenoids, though vital for the baseplate $\mathrm{BChl} a$, are dispensable for BChle. 


\section{INTRODUCTION}

In green photosynthetic bacteria, light-harvesting is carried out by chlorosomes, oblong bodies attached to the inner side of the cytoplasmic membrane of cells $(1,2)$. Chlorosomes consist of highly aggregated bacteriochlorophyll (BChl) $\dagger x$ (hereafter abbreviated as $\mathrm{BChl} x$, with $x$ standing for $c, d$, or $e$ depending on the species), smaller amounts of monomeric BChla and carotenoid (Car) chromophores. The molecular structures of BChlx are such that BChlx$\mathrm{BChl} x$ interactions alone suffice for a spontaneous organization of protein-free aggregates $(3,4)$. Detailed models of the supramolecular arrangement of $\mathrm{BChl} x(5,6)$ match the $5 \mathrm{~nm}$ or $10 \mathrm{~nm}$ diameter of the rod-like structures observed in electron micrographs of chlorosomes from Chloroflexus (Cfl.) aurantiacus and Chlorobium (Chl.) limicola, respectively (7).

$\mathrm{BChl} a$, in contrast to $\mathrm{BChl} x$, is thought to be associated with a chlorosomal protein, denoted CsmA, and to form part of the so-called baseplate on the cytoplasmic membrane side (8). Car molecules in chlorosomes are supposed to participate in photoprotection, mainly by quenching the electronic excitation of $\mathrm{BChl}^{\dagger}$, a $\mathrm{BChl}$ molecule in the lowest triplet state $(9,10)$, to function as light harvesting pigments (11-13), and to play a structural role $(14,15)$. Spectroscopic investigations, based on nanosecond laser photolysis (16) and optically detected magnetic resonance (17), have indicated that Car's photoprotect BChla rather than $\mathrm{BChl} x$; no evidence was found for triplet-triplet energy transfer from BChl $x^{\dagger}$ to the Car ground state $\left(\mathrm{Car}^{0}\right)$, or for a long-lived $\mathrm{BChl} x^{\dagger}$. These results imply that Car might be close to the baseplate to photoprotect the vulnerable BChla monomers.

In chlorosomes the BChl $x$ /Car stoichiometric ratio shows a large variability from $\sim 4 / 1$ to $20 / 1$ depending on species, light regime and growth conditions $(7,18-20)$. This would be a very unfavorable situation if photoprotection in chlorosomes were based exclusively on triplet- 
triplet energy transfer from $\mathrm{BChl} x$ to Car. When the experimental evidence concerning the photophysical behavior of aggregated BChlx is examined (see below), one is led to conclude that BChlx itself does not really need any protection—-because very few triplets are formed in the first place (singlet energy transfer to BChla being very much faster than intersystem crossing), and the few triplets which are produced undergo rapid deactivation (presumably as a result of triplet-triplet migration and subsequent bimolecular events such as annihilation or quenching).

A fluorescence lifetime of a few ns has been determined for $\mathrm{BChl} c$ or BChld monomers $(21,22)$; however, the lifetime of BChlc or BChld is quenched to a few hundreds or a few tens of ps as the BChlx aggregation state changes to dimers or oligomers, respectively $(22,23)$. In chlorosomes or artificial monogalactosyl diglyceride (MGDG) micelles, the fluorescence lifetime of BChlc aggregates is quenched to $40-50$ ps or 9-12 ps under reducing or nonreducing conditions respectively, suggesting in addition that the redox state of $\mathrm{BChl} x$ or quinones could control the flux of excitation energy in chlorosomes $(24,25)$. Interestingly, the fluorescence lifetime of $\mathrm{BChl} x$ in chlorosomes remains quenched, even when energy transfer from BChl $x$ to BChl $a$ is blocked. The perturbation of chlorosomes by $n$-hexanol leads to a blue shift of the BChl $x Q_{\mathrm{y}}$ band that resembles that of truly BChl $x$ monomers; however, fluorescence from the BChlx in the hexanol-perturbed chlorosomes is still very small in comparison to that from true monomers in an organic solvent or a buffer containing Triton X100 (TX) (26). The fluorescence lifetime of BChlc in hexanol-perturbed chlorosomes from Cfl. aurantiacus only increases from 15 ps to 24 ps, despite a large decrease (from $58 \%$ to $13 \%$ ) in the efficiency of energy transfer from BChlc-to-BChla (27). Similarly, alkalinetreated chlorosomes, which are characterized by a selective degradation of the baseplate $\mathrm{BChl} a$, do not show any substantial increase in the fluorescence lifetime of BChl $x$ in 
chlorosomes from Chl. phaeobacteroides or Chl. tepidum (28). To explain the shortening of the fluorescence lifetime of $\mathrm{BChl} x$ in aggregated systems, the former authors have proposed a rapid deactivation channel from the first excited singlet state to the ground state of BChl $x$. Using essentially the same reasoning, Krasnovsky and co-workers had already suggested earlier that formation of $\mathrm{BChl}^{\dagger}$ and singlet oxygen, $\mathrm{O}_{2}\left({ }^{1} \Delta_{\mathrm{g}}\right)$, are suppressed in artificial aggregates or chlorosomes containing BChld because the excitation is preferentially diverted to some rapid non-radiative path which can outcompete intersystem crossing (29).

In a recent publication (16), we investigated the formation of $\mathrm{BChl} e^{\dagger}$ within artificial aggregates and chlorosomes from Chl. phaeobacteroides, and concluded, upon finding no evidence for signals originating from BChle $e^{\dagger}$ (in oxygen-free systems) and no $\mathrm{O}_{2}\left({ }^{1} \Delta_{\mathrm{g}}\right)$ phosphoresence (under aerobic conditions), that BChle in densely packed assemblies is far less liable to photooxidation than monomeric BChle. These investigations have now been extended to hexanol-perturbed chlorosomes, and the results obtained are presented in this paper. Though BChle triplets are seen in hexanol-treated chlorosomes, they are rather shortlived. By comparing nanosecond laser-induced changes in the absorbance of BChlecontaining samples and the time-resolved and steady state measurements of BChle-sensitized $\mathrm{O}_{2}\left({ }^{1} \Delta_{\mathrm{g}}\right)$, we draw some conclusions which agree with those reached by examining untreated chlorosomes, concerning the photoprotection mechanisms present in chlorosomes and other aggregates of BChlx.

\section{MATERIALS AND METHODS}

Growth conditions and chlorosome isolation. Chl. phaeobacteroides strain CL1401 was grown in standard Pfennig mineral medium (30). Chlorosomes from Chl. phaeobacteroides 
were isolated on a $20-50 \%(\mathrm{w} / \mathrm{v})$ sucrose gradient prepared in $50 \mathrm{~m} M$ Tris- $\mathrm{HCl} \mathrm{pH} 8.0$ and 2 $M \mathrm{NaSCN}$ and further purified using a flotation sucrose gradient (31).

Chlorosome treatments and artificial aggregates. Hexanol-perturbed chlorosomes were obtained as described by (26). An aliquot of $10 \mu \mathrm{L}$ of chlorosomes $\left(A_{715}=30\right.$, where $A_{715}$ is the absorbance, corresponding to a path length of $1 \mathrm{~cm}$, for light of wavelength $715 \mathrm{~nm}$ ) were suspended in $2 \mathrm{~mL}$ of $50 \mathrm{~m} M$ Tris- $\mathrm{HCl} \mathrm{pH} 8.0$ saturated with $n$-hexanol $(0.8 \%$, v/v). To reverse the hexanol effect, a stream of $\mathrm{N}_{2}$ was directed at the sample surface to remove $n$ hexanol. Irreversible disruption of chlorosomes was effected by making the chlorosome solution $0.5 \%(\mathrm{w} / \mathrm{v})$ in Triton X-100 (TX). Artificial aggregates of BChle were prepared as described in (32). The second main homologue of BChle containing both the $3^{1}$-R-8-propyl12-ethyl BChle and $3^{1}$-S-8-propyl-12-ethyl BChle was HPLC-purified, harvested in one single pool and dried under a stream of $\mathrm{N}_{2}$ according to (20). MGDG was about $0.003 \%(\mathrm{w} / \mathrm{v})$ and the BChle concentration was $12-16 \mu M$ in $50 \mathrm{mM}$ Tris- $\mathrm{HCl} \mathrm{pH}$ 8.0. Prior to any measurement, artificial aggregates of BChle were incubated at room temperature at least for $20 \mathrm{~min}$ in order to reach a steady absorption spectrum.

Absorption spectroscopy. Absorption spectra of chlorosome preparations were recorded by using a commercial scanning spectrophotometer (Perkin-Elmer Lambda 40). The absorbance values were sampled every $1.0 \mathrm{~nm}$.

Time-resolved triplet-minus-singlet (TmS) spectroscopy. Laser-induced changes in the absorbance of a sample were recorded using right-angle geometry and a gated multi-channel instrument, which has already been described in sufficient detail (16 and reference therein). The gate width, $\Delta t$, was $20 \mathrm{~ns}$ for the measurements whose results are reported here. Let 
$A(\lambda)$ be the absorbance of the sample when all molecules are in their electronic ground states, and $\tilde{A}(\lambda ; t)$ the time-dependent absorbance (at time $t$ ) after the sample has been excited by the laser pulse at $t=0$. The laser induced change in the absorbance of the sample, $\Delta A(\lambda ; t) \equiv \widetilde{A}(\lambda ; t)-A(\lambda)=\log [I(\lambda) / \widetilde{I}(\lambda ; t)]$, was deduced from the measured values of $I(\lambda)$, the detector signal generated by the light emerging from the unirradiated sample, and $\tilde{I}(\lambda ; t)$, the corresponding signal at time $t$. The excitation pulse, which had a duration of about $7 \mathrm{~ns}$ and an energy of 5-10 mJ, was delivered by an optical parametric oscillator (OPO) pumped by the third harmonic of a Nd:YAG laser. A xenon arc lamp $(250 \mathrm{~W})$ and a mechanical chopper $(10 \mathrm{~Hz})$ provided the analyzing beam. For other details, the reader is referred to our earlier publication (16).

Time-resolved and steady state $\mathrm{O}_{2}\left({ }^{1} \Delta_{\mathrm{g}}\right)$ measurements. $\mathrm{O}_{2}\left({ }^{1} \Delta_{\mathrm{g}}\right)$ phosphorescence was studied in air-saturated solutions of BChle at room temperature. Photoexcitation of the sensitizer was achieved by using the same source (OPO) as that used for TmS measurements. Methylene blue (MB) was used as standard to determine the absolute quantum yield of $\mathrm{O}_{2}\left({ }^{1} \Delta_{\mathrm{g}}\right)$ formation $\left(\Phi_{\Delta}\right)$ because its long wavelength absorption peak lies close to that of monomeric BChle. The light leaving the sample (the desired phosphorescence emission as well as unwanted contamination), viewed at a right-angle to the direction of excitation beam, was focussed, with the aid of a convex lens and a parabolic mirror, on the photocathode of the photomultiplier tube (Hamamatsu R5509-42). A long-pass filter (cut-off wavelength $720 \mathrm{~nm}$ ) and a band pass interference filter (centre wavelength $1270 \mathrm{~nm}$ ), placed between the collecting lens and the photomultiplier, served to minimise the intensity of unwanted radiation reaching the detector. The output of the photomultiplier, converted to a voltage by using an 
anode load resistor of $50 \mathrm{ohms,} \mathrm{was} \mathrm{fed} \mathrm{to} \mathrm{a} \mathrm{digital} \mathrm{oscilloscope} \mathrm{(LeCroy} \mathrm{9410)} \mathrm{with} \mathrm{an} \mathrm{input}$ impedance of $50 \mathrm{ohms}$.

Steady state measurements of $\mathrm{O}_{2}\left({ }^{1} \Delta_{\mathrm{g}}\right)$ phosphorescence were carried out using a custom-made commercial instrument (Spex Fluorolog 3-2-2-Triax 320). The excitation channel is of standard configuration, containing a xenon arc lamp and a double monochromator (with 1200 lines/mm gratings blazed at $500 \mathrm{~nm}$ ). The emission channel has two independent detection systems, one of which is used for detecting emission in the near infrared region (800-1600 $\mathrm{nm}$ ). Briefly, this system consists of a dual-purpose dispersing device (Triax 320 with two gratings, both blazed at $1000 \mathrm{~nm}$, one with 150 lines/mm and the other with 300 lines), which directs the dispersed light to a narrow slit (monochromator mode) or a wide opening (spectrograph mode). In the former case, the near infrared photomultiplier (R5509-42), and in the latter case, a thermoelectrically cooled InGaAs-array (with 256 elements) is used as the detector. The results described in this paper were obtained by using the instrument in the spectrograph mode and selecting the 300 lines/mm grating.

\section{RESULTS AND DISCUSSION}

We will begin by comparing the absorption spectra of intact chlorosomes with those of hexanol-treated chlorosomes and TX-disrupted chlorosomes; the TmS spectra of the last two systems will be examined next, and used for analyzing, in the final part, data concerning the sensitization (and quenching) of $\mathrm{O}_{2}\left({ }^{1} \Delta_{\mathrm{g}}\right)$ by monomeric $\mathrm{BChl} e^{\dagger}$ (and $\mathrm{BChl} e^{0}$, respectively).

\section{Absorption spectra of treated chlorosomes}


When isolated chlorosomes are treated with hexanol-saturated buffer $(0.8 \% n$-hexanol, v/v), the absorption spectrum is replaced by a spectrum that resembles that of the $\mathrm{BChl} x$ monomer (26). An absorption spectrum close to that of the original, untreated chlorosomes is obtained if one dilutes, by a factor of two or more, the treated chlorosomes with the hexanol-free buffer. We chose evaporation as a means of lowering the hexanol content of treated chlorosomes; this was achieved, as already stated in the Materials and Methods section, by directing a stream of gaseous $\mathrm{N}_{2}$ at the surface of the sample. Figure 1 displays how the absorption spectrum changes, with progressive loss of $n$-hexanol, from a shape typical of hexanol-treated chlorosomes to one that no longer changes with time, and may therefore be identified with hexanol-free, or "re-cycled" chlorosomes. The inset provides a comparison of the absorption spectrum of untreated chlorosomes with that of the final spectrum in the set; the $Q_{\mathrm{y}}$ peak of the re-cycled chlorosomes is blue-shifted by about $10 \mathrm{~nm}$ with respect to the corresponding peak of the untreated chlorosomes. It has been reported that reversal of hexanol treatment of Chl. limicola chlorosomes leads to a red shift of $2 \mathrm{~nm}$ or a blue shift of $8 \mathrm{~nm}$ depending on whether the dilution is performed slowly or rapidly (26). Also, it has been found that hexanoltreated chlorosomes of $C f l$. aurantiacus do not return to the original shape or size after twofold dilution even though most of the spectroscopic properties (as judged from steady-state absorption and time-resolved fluorescence studies) are restored by the dilution (27).

Before returning to the spectra of our untreated and re-cycled chlorosomes, we pause to recall that the $Q_{\mathrm{y}}$ peak of carotenoid-depeleted chlorosomes (of Chl. phaeobacteroides) is also blue-shifted by $10 \mathrm{~nm}$ relative to that of native chlorosomes (33). This blue shift, and some other differences (a two-fold increase in the CD signal of the carotenoid-depleted chlorosomes and an appreciable reduction in the magnitude of the fluorescence anisotropy), were explained in terms of a difference in the organization of the chromophores in the two 
systems, particularly the angle between the direction of the $Q_{\mathrm{y}}$ transition moment of BChle and the axis of the rod. The blue shift resulting from the addition-and-removal of $n$-hexanol is most probably due to a reorganization of the pigments; measurements of $\mathrm{CD}$ and fluorescence anisotropy are needed to see whether the pigment organization in re-cycled chlorosomes is similar to that in carotenoid-depleted chlorosomes.

A noteworthy feature, made manifest by the presence of several isosbestic points in Fig. 1, is that the spectra are internally linear (34); that is to say, any spectrum of the set can be reconstructed by forming a linear combination of two other members of the set. It is also worth observing that the total area under each spectrum remains practically unchanged.

Though hexanol treatment leads to an absorption spectrum which appears, at least at first sight, to be "monomeric", the BChle pigments remain densely packed in chlorosomal vesicles. Indeed, the fluorescence of this apparently monomeric form is highly quenched compared to that from a truly monomeric solution in an organic solvent $(27,35)$. The $\mathrm{CD}$ signal of chlorosomes and artificial aggregates becomes exceedingly weak after hexanol treatment, and contains no hint of excitonic interactions $(35,36)$. Given the absence of such interactions and a high local concentration of pigments, one might expect to see signs of flattening (caused by shadowing) in the absorption spectrum of the hexanol-perturbed chlorosomes (37). Differences in the absorption spectra of hexanol-treated chlorosomes and TX-disrupted chlorosomes are therefore worth investigating.

When TX is added to a suspension of untreated chlorosomes, the absorption spectrum gradually changes, resembling finally the absorption spectrum of a pigment extract of chlorosomes in an organic solvent. A set of spectra obtained in this manner is plotted in Fig. 
2; the spectra in this set are, like those in Fig. 1, internally linear and are all of roughly the same area. The final spectrum in Fig. 2 is close to the absorption spectrum of a pigment extract in ethanol (20), and will henceforth be identified with a system where all pigmentpigment interactions have been made negligible. On comparing the final spectrum in Fig. 2 with the spectrum of hexanol-treated chlorosomes (the initial spectrum in Fig. 1), one sees that the peaks in the hexanol-treated chlorosomes are appreciably broader than those in the true monomeric spectrum (Fig. 2 inset). We have applied Duysen's treatment and verified that shadowing cannot account for the observed differences (data not shown). We ascribe the broadening and bathochromic shift seen in the absorption spectrum of hexanol-treated chlorosome to dispersion interactions, which cause an overall solvent-induced shift to longer wavelengths; a similar explanation was offered for the spectral properties of the lightharvesting complex LHCII of the chlorophyll $a / c$ containing yellow-green alga Pleurochloris meiringensis (38).

\section{TmS spectra}

The difference between TX-disrupted chlorosomes and hexanol-treated chlorosomes becomes more noticeable when one compares the TmS spectra of the two systems, which are shown in Figs. 3 and 4, respectively. Laser irradiation of the former system gives rise to absorbance changes (Fig. 3) which can be identified with the TmS spectrum of true BChle monomers; the spectrum is dominated by negative signals which reflect loss of ground-state BChle molecules $\left(\mathrm{BChl} e^{0}\right)$. The relatively long lifetime of the BChl $e^{\dagger}$ signal, and the absence of a TmS signal attributable to Car shows that bimolecular routes for deactivation (triplet-triplet annihilation and quenching by Car or oxygen) have been suppressed by confining the pigments to a micellar environment. 
Whereas the spectra in Fig. 3 contain no discernible contribution from $\mathrm{Car}^{\dagger}$, the dominant feature of the corresponding spectra of hexanol-treated chlorosomes (Fig. 4) is the TmS spectrum of Car, which is known to have a positive peak around $520 \mathrm{~nm}\left(T_{\mathrm{n}} \leftarrow T_{1}\right.$ absorption) in almost all photosynthetic specimens $(16,38-40)$. To unravel the three spectra in Fig. 4, it is helpful to observe that the $520 \mathrm{~nm}$ peak in $\Delta_{1}(\lambda)$ (delay $20 \mathrm{~ns}$ ) is lower than that in $\Delta_{2}(\lambda)$ (delay $200 \mathrm{~ns}$ ), and to note that a plot (shown in Fig. 5) of the difference $\Delta_{0}(\lambda) \equiv \Delta_{1}(\lambda)-\alpha \Delta_{2}(\lambda)$ with $\alpha$ a positive constant slightly smaller than unity, can be made to resemble the TmS spectra appearing in Fig. 3. Accordingly, $\Delta_{0}(\lambda)$ can be viewed as the TmS spectrum (at the shortest delay) of free $\mathrm{BChl} e^{\dagger}$ in hexanol-perturbed chlorosomes, and $\alpha \Delta_{2}(\lambda)$ as the TmS spectrum (again at the shortest delay) of Car in hexanol-perturbed chlorosomes. The fact that $\Delta A(\lambda ; t)$ changes, within $200 \mathrm{~ns}$, from $\Delta_{1}(\lambda)=\Delta_{0}(\lambda)+\alpha \Delta_{2}(\lambda)$ to $\Delta_{2}(\lambda)$ carries two implications: First, the lifetime of free BChl $e^{\dagger}$ is rather short, and secondly, only a small fraction of these triplets transfer their electronic excitation to a Car. The second conclusion is derived from the following considerations: Since the absorption coefficients of the BChle Soret peak (which dominantes the TmS signal) and the Car $T_{\mathrm{n}} \leftarrow T_{1}$ peak are nearly equal (ca.1.5 $\left.\times 10^{-1} \mathrm{M}^{-1} \mathrm{~cm}^{-1}\right)(20,41)$, energy transfer from BChl $e^{\dagger}$ to Car would cause a recovery of $\mathrm{BChl} e^{0}$, and thereby a reduction in the magnitude of the negative TmS peak in $\Delta_{0}(\lambda)$ at the wavelength of the Soret peak accompanied by a comparable increase in the height of the Car peak. However, the increase in the height of the latter peak is much smaller than the decrease in the former peak. Evidently, the bulk of the free BChle triplets are not quenched by Car, and must decay through some other nonradiative channel (presumably triplet-triplet annihilation). At delays longer than $200 \mathrm{~ns}$, the shape of the TmS spectrum of hexanol-treated chlorosomes does not change; on referring to Fig. 4, one sees that this is 
borne out by the identical shapes of $\Delta_{2}(\lambda)$ and $\Delta_{3}(\lambda)$. This means that the small negative signal in the red region in $\Delta_{2}(\lambda)$ and $\Delta_{3}(\lambda)$ reflects the perturbation, by a $\operatorname{Car}^{\dagger}$, of the absorption spectrum of an adjacent $\mathrm{BChl} e^{0}$; since enough has been said about this perturbation in our earlier work (16), we refrain from elaborating on this point here.

We would now like to compare the main features of the TmS spectra of untreated chlorosomes (16) and hexanol-treated ones (this study). Perhaps the most important difference is the absence (in untreated chlorosomes) and presence (in hexanol-treated chlorosomes) of a TmS signal contributed by BChle $e^{\dagger}$. Moreover, the TmS signal of Car, present in both cases, becomes about three-fold larger after hexanol treatment (Fig. 6). Taken together, the two differences imply that, other things being equal, more triplets are produced in hexanol-treated chlorosomes. In untreated chlorosomes, the efficiency of singlet energy transfer from BChle to BChla is rather high $(27,42)$ and all BChl triplet donors (to Car acceptors) appear to be monomeric BChl $a^{\dagger}$. Since the singlet transfer efficiency suffers a large reduction after hexanol treatment (27), our results can only be explained if most triplets are now monomeric $\mathrm{BChl} e^{\dagger}$ (otherwise the initial TmS signal of Car would have been very much lower). In the light of the foregoing conclusion, it also becomes relevant to compare the shapes of the TmS spectra of Car in untreated and hexanol-treated chlorosomes. Figure 6 shows that the two spectra differ appreciably. The TmS signal in untreated chlorosomes is contributed by those Car which are in contact with BChl $a^{\dagger}$ donors $(16,17)$, whereas that in hexanol-treated chlorosomes originates, it has been argued above, largely from the $\operatorname{Car}^{0}$ acceptors which are in contact with BChl $e^{\dagger}$ donors. The fact that there are about 15-20 Car molecules per BChla in chlorosomes from Chl. phaeobacteriodes (20) makes one suspect that not all Car molecules have a BChla neighbour; if one accepts this conclusion, the difference between the two spectra reflects a dissimilarity in the environments of the acceptors in the two systems. One 
could argue that difference in the two TmS spectra is simply a consequence of the perturbation in the environment of a single Car pool by the addition of $n$-hexanol; it seems more likely that the Car pool is heterogeneous in both types of chlorosomes, and the organization of these pools is subject to the presence or absence of $n$-hexanol.

\section{Singlet oxygen photogeneration and quenching by BChle monomers}

BChle was dissolved in air-saturated acetone in a range of concentrations between $0.5-10 \mu M$ to investigate the sensitization of singlet oxygen, $\mathrm{O}_{2}\left({ }^{1} \Delta_{\mathrm{g}}\right)$. Acetone was chosen because the reported lifetime of $\mathrm{O}_{2}\left({ }^{1} \Delta_{\mathrm{g}}\right)$ in this solvent is rather long, around $50 \mu \mathrm{s}$ (43), and also because we wanted to compare our results with those obtained by (44), in which $\mathrm{O}_{2}\left({ }^{1} \Delta_{\mathrm{g}}\right)$ sensitization by BChlc and BChld in acetone was measured. The $1270 \mathrm{~nm}$ phosphorescence emission of $\mathrm{O}_{2}\left({ }^{1} \Delta_{\mathrm{g}}\right)$ was detected when BChle was excited at $475 \mathrm{~nm}$ and disappeared when oxygen was removed by bubbling Ar through the sample. Since we were also able to detect, under oxygen-free conditions, a long-lived (a few tens of $\mu \mathrm{s}$ ) TmS spectrum of BChle (not shown), it is reasonable to conclude that $\mathrm{O}_{2}\left({ }^{1} \Delta_{\mathrm{g}}\right)$ was sensitized by BChl $e^{\dagger}$. The decay rate of the $\mathrm{O}_{2}\left({ }^{1} \Delta_{\mathrm{g}}\right)$ phosphorescence emission signal was found to depend on the BChle concentration (Fig. 7). A plot of $k([Q])$, the rate constant for overall decay, against $[Q]$, the concentration of the quencher (in this case BChle), turns out to be linear, showing that one may write $k([Q])=k_{0}+k_{q}[Q]$. The plot, shown in the inset of Fig. 7, leads to the following values: $k_{0}=(18.5 \pm 0.7) \times 10^{3} \mathrm{~s}^{-1}$ and $k_{q}=(1.4 \pm 0.1) \times 10^{9} \mathrm{M}^{-1} \mathrm{~s}^{-1}$. Our result for $k_{0}$ implies a lifetime of $54 \mu \mathrm{s}$, in good agreement with recent literature values (43); the value for $k_{\mathrm{q}}$ is close to those determined for the quenching of $\mathrm{O}_{2}\left({ }^{1} \Delta_{\mathrm{g}}\right)$ by BChl $c^{0}$ and BChld $d^{0}$, the ground state BChlc and BChld molecules, by Krasnovsky and co-authors (44), who pointed out BChl $c^{0}$ and $\mathrm{BChl} d^{0}$ 
are more efficient physical quenchers of $\mathrm{O}_{2}\left({ }^{1} \Delta_{\mathrm{g}}\right)$ than $\mathrm{Chl} a$. The results reported here show that their conclusion applies to all three main chlorosomal $\mathrm{BChl} x^{0}$ present in green photosynthetic bacteria. As judged from some changes in the absorption spectrum of BChle in air-saturated acetone and the concomitant formation of photooxidized BChle derivatives (not identified in this study), the quenching of $\mathrm{O}_{2}\left({ }^{1} \Delta_{\mathrm{g}}\right)$ by BChle $e^{0}$ must be due at least partly to a chemical reaction.

The absolute quantum yield of $\mathrm{O}_{2}\left({ }^{1} \Delta_{\mathrm{g}}\right)$ formation $\left(\Phi_{\Delta}\right)$ by BChle monomers in airsaturated acetone was determined by comparing the intensity of the steady state $\mathrm{O}_{2}\left({ }^{1} \Delta_{\mathrm{g}}\right)$ phosphorescence in a solution containing BChle with that in a solution of $\mathrm{MB}$, under conditions where excitation wavelength, sample absorbance, and detection sensitivity were identical. $\Phi_{\Delta}$ for MB in acetone was taken as 0.5. Since BChle $e^{0}$ was found to quench $\mathrm{O}_{2}\left({ }^{1} \Delta_{\mathrm{g}}\right)$, BChle concentration was kept lower than $1 \mu M$ in these experiments. The obtained value for $\Phi_{\Delta}$ was $0.65 \pm 0.15$; the corresponding values for BChlc and BChld have been reported to be 0.61 and 0.55 , respectively (44).

\section{Singlet oxygen photogeneration by BChle aggregates}

In our previous publication (16), we reported our failure to detect time-resolved $\mathrm{O}_{2}\left({ }^{1} \Delta_{\mathrm{g}}\right)$ emission from untreated chlorosomes and artificial aggregates containing BChle; with this equipment we also failed to observe any $1270 \mathrm{~nm}$ phosophorescence from samples containing hexanol-treated chlorosomes. Attempts were therefore made to detect steady state $\mathrm{O}_{2}\left({ }^{1} \Delta_{\mathrm{g}}\right)$ phosphorescence in several preparations of BChle aggregates, using the steady-state equipment which became available afterwards. None of the three systems listed above gave a positive result. The simplest system, artificial MGDG aggregates of BChle, yielded no 
$\mathrm{O}_{2}\left({ }^{1} \Delta_{\mathrm{g}}\right)$ phosporescence upon excitation at $704 \mathrm{~nm}$ (Fig. 8). MGDG might be suspected to act as a physical barrier obstructing the entry of $\mathrm{O}_{2}\left({ }^{3} \Sigma_{\mathrm{g}}{ }^{-}\right)$inside the artificial MGDG aggregates; however, the fact that even aggregates of BChle in hexane did not show any $\mathrm{O}_{2}\left({ }^{1} \Delta_{\mathrm{g}}\right)$ phosphorescence (data not shown) suggests that in both aqueous and organic solutions the absence of $\mathrm{O}_{2}\left({ }^{1} \Delta_{\mathrm{g}}\right)$ signal by BChle aggregates reflects the low yield for BChl $e^{\dagger}$ formation (16), caused by the rapid non-radiative deactivation channel of excitation. Our results corroborate the findings of Krasnovsky and co-workers (44), who reported no $\mathrm{O}_{2}\left({ }^{1} \Delta_{\mathrm{g}}\right)$ photogeneration in aggregate systems of BChld.

With a view to illustrating the detection capability of our detection equipment, we decided to enhance the yield of $\mathrm{BChl} e^{\dagger}$ formation by disrupting artificial MGDG aggregates of BChle with $0.5 \%$ TX. The shape and the lifetime of the $\Delta A(\lambda ; \mathrm{t})$ spectrum of TX-disrupted artificial aggregates of BChle (not shown) turned out to be practically indistinguishable from that of TX-disrupted chlorosomes, with a large, long-lived signal ascribable to the BChle TmS spectrum. As a result of the long lifetime of BChl $e^{\dagger}$ in TX micelles, $\mathrm{O}_{2}\left({ }^{1} \Delta_{\mathrm{g}}\right)$ phosphorescence could now be observed as a hump (peaking at $1270 \mathrm{~nm}$ ) superposed on the fluorescence emission background of BChle (Fig. 8); when oxygen was purged from the system (by blowing Ar at the surface of the solution for 15 minutes), $\mathrm{O}_{2}\left({ }^{1} \Delta_{\mathrm{g}}\right)$ phosphorescence could not be observed any longer. As is to be exptected, the background fluorescence emission of BChle in artificial MGDG aggregates became larger after disruption by TX. It should be noted that this is a more severe test of the apparatus than recording $\mathrm{O}_{2}\left({ }^{1} \Delta_{\mathrm{g}}\right)$ emission from an acetone solution of BChle, since the phosphorescence yield was found to depend on the TX concentration used for disrupting the artificial MGDG aggregates (data not shown). The low BChle $e^{\dagger}$ yield in native chlorosomes (16), the sub- $\mu$ s quenching of $\mathrm{BChl} e^{\dagger}$ by Car and $\mathrm{BChl} e^{0}$ in hexanol-perturbed, rather than lack of adequate detection 
sensitivity, are the principal reasons for our persistent failure to find some evidence of $\mathrm{O}_{2}\left({ }^{1} \Delta_{\mathrm{g}}\right)$ photogeneration in these aggregate systems.

\section{CONCLUSIONS}

In conclusion, we propose that chlorosomes utilise two photoprotection mechanisms. In one Car plays the main role, quenching $\mathrm{BChl}^{\dagger}$ through triplet-triplet energy transfer and forming $\mathrm{Car}^{\dagger}$, which is able to dissiapte its energy in the form of heat in a harmless manner. This mechanism forestalls sensitization of $\mathrm{O}_{2}\left({ }^{1} \Delta_{\mathrm{g}}\right)$, triplet-triplet energy transfer from $\mathrm{BChl}^{\dagger}$ to an adjacent $\mathrm{Car}^{0}$ being faster than quenching of $\mathrm{BChl}^{\dagger}$ by $\mathrm{O}_{2}\left({ }^{3} \Sigma_{\mathrm{g}}{ }^{-}\right)$, which requires molecular diffusion; it accounts for the photoprotection of BChla monomers situated in the chlorosome baseplate. The other, which may be called a self-protection mechanism, relies on shortening the lifetimes of excited states, singlet as well as triplet; here $\mathrm{O}_{2}\left({ }^{1} \Delta_{\mathrm{g}}\right)$ sensitization does not pose a serious threat because the initial yield of $\mathrm{BChl}^{\dagger}$ is kept low, and most of the triplets, once formed, are deactivated, even in the absence of Car, before they encounter ground-state $\mathrm{O}_{2}$.

Acknowledgements. We acknowledge financial support from the Research Council of Norway and the European Union (contract FMRX-CT96-0081). 


\section{REFERENCES}

1. Blankenship, R. E., J. M. Olson and M. Miller (1995) Antenna complexes from green photosynthetic bacteria. In Anoxygenic Photosynthetic Bacteria (Edited by R. E. Blankenship, M. T. Madigan and C. E. Bauer), pp. 399-435. Kluwer Academic Publishers, Dordrecht, The Netherlands.

2. Olson, J. M. (1998) Chlorosome organization and function in green photosynthetic bacteria. Photochem. Photobiol. 67, 61-75.

3. Miller, M., T. Gillbro and J. M. Olson (1993) Aqueous aggregates of bacteriochlorophyll $c$ as a model for pigment organization in chlorosomes. Photochem. Photobiol. 57, 98-102.

4. Holzwarth, A. R., K. Griebenow and K. Schaffner (1990) A photosynthetic antenna system which contains a protein-free chromophore aggregate. Z. Naturforsch. 45, 203296.

5. Holzwarth, A. R. and K. Schaffner (1994) On the structure of bacteriochlorophyll molecular aggregates in the chlorosomes of green bacteria. A molecular modelling study. Photosynth. Res. 41, 225-233.

6. Steensgaard, D. B., H. Wackerbarth, P. Hildebrandt and A. R. Holzwarth (2000) Diastereoselective control of bacteriochlorophyll $e$ aggregation. $3^{1}$-S-BChl $e$ is essential for the formation of chlorosome-like aggregates. J. Phys. Chem. B 104, 10379-10386. 
7. Oelze, J. and J. R. Golecki (1995) Membranes and chlorosomes of green bacteria: Structure, composition and development. In Anoxygenic Photosynthetic Bacteria (Edited by R. E. Blankenship, M. T. Madigan and C. E. Bauer), pp. 259-278. Kluwer Academic Publishers, Dordrecht, The Netherlands.

8. Sakuragi, Y., N. U. Frigaard, K. Shimada and K. Matsuura (1999) Association of bacteriochlorophyll $a$ with the CsmA protein in chlorosomes of the photosynthetic green filamentous bacterium Chloroflexus aurantiacus. Biochim. Biophys. Acta 1413, 172-180.

9. Psencik, J., G. F. W. Searle, J. Hala and T. J. Schaafsma (1994) Fluorescence detected magnetic resonance (FDMR) of green sulfur photosynthetic bacteria Chlorobium sp. Photosynth. Res. 40, 1-10.

10. Psencik, J., T. J. Schaafsma, G. F. W. Searle and J. Hala (1997) Fluorescence detected magnetic resonance of monomers and aggregates of bacteriochlorophylls of green sulfur bacteria Chlorobium sp. Photosynth. Res. 52, 83-92.

11. van Dorssen, R. J., H. Vasmel and J. Amesz (1986) Pigment organization and energy transfer in the green photosynthetic bacterium Chloroflexus aurantiacus. II. The chlorosome. Photosynth. Res. 9, 33-45.

12. Otte S. C. M., J. C. van der Heiden, N. Pfennig and J. Amesz (1991) A comparative study of the optical characteristics of intact cells of photosynthetic green sulfur bacteria containing bacteriochlorophyll $c, d$ or e. Photosynth. Res. 28, 77-87. 
13. Melø, T. B., N. U. Frigaard, K. Matsuura and K. R. Naqvi (2000) Electronic energy transfer involving carotenoid pigments in chlorosomes of two green bacteria: Chlorobium tepidum and Chloroflexus auranticus. Spectrochim. Acta A 56, 2001-2010.

14. Arellano, J. B., C. M. Borrego, A. Martínez-Planells and L. J. Garcia-Gil (2001) Effect of carotenoid deficiency on cells and chlorosomes from Chlorobium phaeobacteroides. Arch. Microbiol. 175, 226-233.

15. Foidl, M., J. R. Golecki and J. Oelze (1997) Phototrophic growth and chlorosome formation in Chloroflexus aurantiacus under conditions of carotenoid deficiency. Photosynth. Res. 54, 219-226.

16. Arellano, J. B., T. B. Melø, C. M. Borrego, J. Garcia-Gil and K. R. Naqvi (2000) Nanosecond laser photolysis studies of chlorosomes and artificial aggregates containing bacteriochlorophyll $e$ : Evidence for the proximity of carotenoids and bacteriochlorophyll $a$ in chlorosomes from Chlorobium phaeobacteroides strain CL1401. Photochem. Photobiol. 72, 669-675.

17. Carbonera, D., E. Bordignon, G. Giacometti, G. Agostini, A. Vianelli and C. Vannini (2001) Fluorescence and absorption detected magnetic resonance of chlorosomes from green bacteria Chlorobium tepidum and Chloroflexus aurantiacus. A comparative study. J. Phys. Chem B 105, 246-255. 
18. Liaaen-Jensen, S. (1965) Bacterial carotenoids. XVIII. Aryl carotenoids from Phaeobium. Acta Chem. Scand. 19, 1025-1030.

19. Schmidt, K. (1980) A comparative study on the composition of chlorosomes (Chlorobium vesicles) and cytoplasmic membranes from Chloroflexus aurantiacus OK70-fl and Chlorobium limicola f. thiosulfatophilum strain 6230. Arch. Microbiol. 124, $21-31$

20. Borrego, C. M., J. B. Arellano, C. A. Abellà, T. Gillbro and L. J. Garcia-Gil (1999) The molar extinction coefficient of bacteriochlorophyll $e$ and the pigment stoichiometry in Chlorobium phaeobacteroides. Photosynth. Res. 60, 257-264.

21. Brune, D. C., R. E. Blankenship and G. R. Seely (1988) Fluorescence quantum yields and lifetimes for bacteriochlorophyll c. Photochem. Photobiol. 47, 759-763.

22. Cheng, P., P. Liddell, X. C. Ma and R. E. Blankenship (1993) Properties of Zn and Mg methyl bacteriopheophorbide- $d$ and their aggregates. Photochem. Photobiol. 58, $290-295$

23. Causgrove, T. P., D. C. Brune, R. E. Blankenship and J. M. Olson (1990) Fluorescence lifetimes of dimers and higher oligomers of bacteriochlorophyll $c$ from Chlorobium limicola. Photosynth. Res. 25, 1-10. 
24. van Noort, P. I., Y. Zhu, R. LoBrutto and R. E. Blankenship (1997) Redox effects on the excited-state lifetime in chlorosomes and bacteriochlorophyll c oligomers. Biophys. J. 72, $316-325$.

25. Frigaard, N. U., S. Takaichi, M. Hirota, K. Shimada and K. Matsuura (1997) Quinones in chlorosomes of green sulfur bacteria and their role in the redox-dependent fluorescence studied in chlorosome-like bacteriochlorophyll $c$ aggregates. Arch. Microbiol. 167, 343349.

26. Matsuura, K. and J. M. Olson (1990) Reversible conversion of aggregated bacteriochlorophyll $c$ to the monomeric form by 1-hexanol in chlorosomes from Chlorobium and Chloroflexus. Biochim. Biophys. Acta 1019, 233-238.

27. Zhu, Y., B. L. Ramakrishna, P. Y. van Noort and R. E. Blankenship (1995) Microscopic and spectroscopic studies of untreated and hexanol-treated chlorosomes from Chloroflexus aurantiacus. Biochim. Biophys. Acta 1232, 197-207.

28. van Walree, C. A., Y. Sakuragi, D. B. Steensgaard, C. S. Bösinger, N.-U. Frigaard, R. P. Cox, A. R. Holzwarth and M. Miller (1999) Effect of alkaline treatment on bacteriochlorophyll $a$, quinones and energy transfer in chlorosomes from Chlorobium tepidum and Chlorobium phaeobacteroides. Photochem. Photobiol. 69, 322-328.

29. Krasnovsky, A. A., J. Lopez, P. Cheng, P. A. Liddell, R. E. Blankenship, T. A. Moore and D. Gust (1994) Generation and quenching of singlet molecular oxygen by aggregated bacteriochlorophyll $d$ in model systems and chlorosomes. Photosynth. Res. 40, 191-198. 
30. Trüper, H. G. and N. Pfennig (1992) The family Chlorobiaceae. In The Prokaryotes. A Handbook on the Biology of Bacteria: Ecophysiology, Isolation, Identification, Applications (Edited by A. Balows, H. G. Trüper, M. Dworkin, W. Harder and K. H. Schleifer), pp. 3583-3592, $2^{\text {nd }}$ edition. Springer-Verlag, Berlin, Germany.

31. Steensgaard, D. B, K. Matsuura, R. P. Cox and M. Miller (1997) Changes in bacteriochlorophyll $c$ organization during acid treatment of chlorosomes from Chlorobium tepidum. Photochem. Photobiol. 65, 129-134.

32. Hirota, M., T. Moriyama, K. Shimada, M. Miller, J. M. Olson and K. Matsuura (1992) High degree of organization of bacteriochlorophyll $c$ in chlorosomes-like aggregates spontaneously assembled in aqueous solution. Biochim. Biophys. Acta 1099, 271-274.

33. Arellano, J. B., J. Psencik, C. M. Borrego, Y. Z. Ma, R. Guyoneaud, J. Garcia-Gil and T. Gillbro (2000) Effect of carotenoid biosynthesis inhibition on the chlorosome organization in Chlorobium phaeobacteroides strain CL1401. Photochem. Photobiol. 71, $715-723$.

34. Brynestad, J. and G. P. Smith (1968) Isosbestic points and internally linear spectra generated by changes in solvent composition or temperature. J. Phys. Chem. 72, 296-300. 
35. Zhu, Y., S. Lin, B. L. Ramakrishna, P. I. van Noort and R. E. Blankenship (1996) Self quenching of chlorosome chlorophylls in water and hexanol-saturated water. Photosynth. Res. 47, 207-218.

36. Wang, Z. Y., G. Marx, M. Umetsu, M. Kobayashi, M. Mimuro and T. Nozawa (1995) Morphology ans spectroscopy of chlorosomes from Chlorobium tepidum by alcohol treatments. Biochim. Biophys. Acta 1232, 187-196.

37. Duysens, L. N. M. (1956) The flattening of the absorption spectrum of suspensions, as compared to that of solutions. Biochim. Biophys. Acta 19, 1-13.

38. Büchel, C., K. R. Naqvi and T. B. Melø (1998) Pigment-pigment interactions in thylakoids and LHCII of chlorophyll a/c containing alga Pleurochloris meiringensis: analysis of fluorescence-excitation and triplet-minus-singlet spectra. Spectrochim. Acta A 54, 719-726.

39. Van der Voss, R. D., D. Carbonera and A. J. Hoff (1991) Microwave and optical spectroscopy of carotenoid triplets in light-harvesting complex LHCII of spinach by absorbance-detected magnetic resonance. App. Magn. Reson. 2, 179-202.

40. Naqvi, K. R., T. B. Melø, B. B. Raju, T. Jávorfi, I. Simidjiev and G. Garab (1997) Quenching of chlorophyll $a$ singlets and triplets by carotenoids in light-harvesting complex of photosystem II: comparison of aggregates with trimers. Spectrochim. Acta A 53, 2659-7667. 
41. Carmichael, I. and G. L. Hug (1986) Triplet-triplet absorption spectra of organic molecules in condensed phases. J. Phys. Chem. Ref. Data 15, 1-250.

42. Psencik, J., Y. M. Ma, J. B. Arellano, J. Garcia-Gil, A. R. Holzwarth and T. Gillbro (2002) Excitation energy transfer in chlorosomes of Chlorobium phaeobacteroides strain CL1401: The role of carotenoids. Photosynth. Res. In press.

43. Wilkinson, F. (1995) Rate constants for the decay and reactions of the lowest electronically excited singlet state of molecular oxygen in solution. An expanded and revised compilation. J. Phys. Chem. Ref. Data 24, 663-1021.

44. Krasnovsky, A. A., P. Cheng, R. E. Blankenship, T. A. Moore and D. Gust (1993) The photophysics of monomeric bacteriochlorophylls- $c$ and bacteriochlorophylls- $d$ and their derivatives: Properties of the triplet-state and singlet oxygen photogeneration and quenching. Photochem. Photobiol. 57, 324-330. 


\section{FIGURE LEGENDS}

Figure 1. Changes in the absorption spectrum of hexanol-perturbed chlorosomes from Chlorobium phaeobacteroides produced by blowing $\mathrm{N}_{2}(\mathrm{~g})$ on the surface of the solution. Arrows indicate increase or decrease in absorbance. Absorption spectra were recorded at intervals of $\sim 2 \mathrm{~min}$. The comparison between re-cycled chlorosomes after removal of $n$ hexanol (solid line) and native chlorosomes (dotted line) is shown in the inset.

Figure 2. Changes in the absorption spectrum of chlorosomes from Chlorobium phaeobacteroides produced by adding $0.5 \%$ Triton X-100. Arrows indicate increase or decrease in absorbance. Absorption spectra were recorded at intervals of $\sim 2 \mathrm{~min}$. The comparison between Triton X-100 disrupted (solid line) and hexanol-perturbed (dotted line) chlorosomes is shown in the inset.

Figure 3. Time-resolved, laser-induced changes, $\Delta A(\lambda ; \mathrm{t})$, in the absorbance of Triton $\mathrm{X}-100$ disrupted chlorosomes at different delays. The initial absorbance was about 0.4 at $650 \mathrm{~nm}$. The inverted absorption (dashed line) of the sample is plotted for better comparison.

Figure 4. Time-resolved, laser-induced changes, $\Delta A(\lambda ; \mathrm{t})$, in the absorbance of hexanolperturbed chlorosomes at different delays. The initial absorbance was about 0.4 at $650 \mathrm{~nm}$. The inverted absorption (dashed line) of the sample is plotted for better comparison.

Figure 5. Comparison between the TmS spectra (at the shortest delay) of free BChle in hexanol-perturbed (dark solid line) and in Triton X-100 disrupted (gray solid line) chlorosomes. The calculated value of $\Delta_{0}(\lambda)$ was obtained as a substraction between $\Delta_{1}(\lambda)$ and 
a fraction close to the unit of $\Delta_{2}(\lambda)$, both TmS spectra shown in Figure 4. The inverted absorption spectrum of hexanol-perturbed chlorosomes is shown as a gray dashed line.

Figure 6. Comparison between the TmS spectra of Carotenoids (both at a delay of $0.7 \mu$ s to avoid any contribution by BChl triplets) in hexanol-perturbed (dark line) and native (open circle + gray line) chlorosomes. The TmS spectrum of carotenoids in native chlorosomes was multiplied by a factor of 3 .

Figure 7. Semilogarithmic plot of singlet oxygen phosphorescence decay in BChle-dissolved acetone solutions. The BChle concentrations were $5.0 \mu M$ (lower most trace), $2.5 \mu M$ (middle trace) and $1.5 \mu M$ (upper most trace). BChle was excited at $475 \mathrm{~nm}$ and each plot was an average of 64 laser flashes. A Stern-Volmer plot of the decay rate of singlet oxygen versus BChle concentration in acetone is shown in the inset.

Figure 8. Steady state infrared emission from artificial MGDG aggregates of BChle (thin solid trace) and Triton X-100 disrupted artificial MGDG aggregates of BChle in air-saturated solution (thick solid trace) or in Ar-saturated solution (opened circle). The initial absorbance of the artificial MGDG BChle aggregates was about $0.8 \mathrm{~cm}^{-1}$ at $704 \mathrm{~nm}$. All the solutions had equal number of BChle molecules. 
Figure 1

Arellano et al

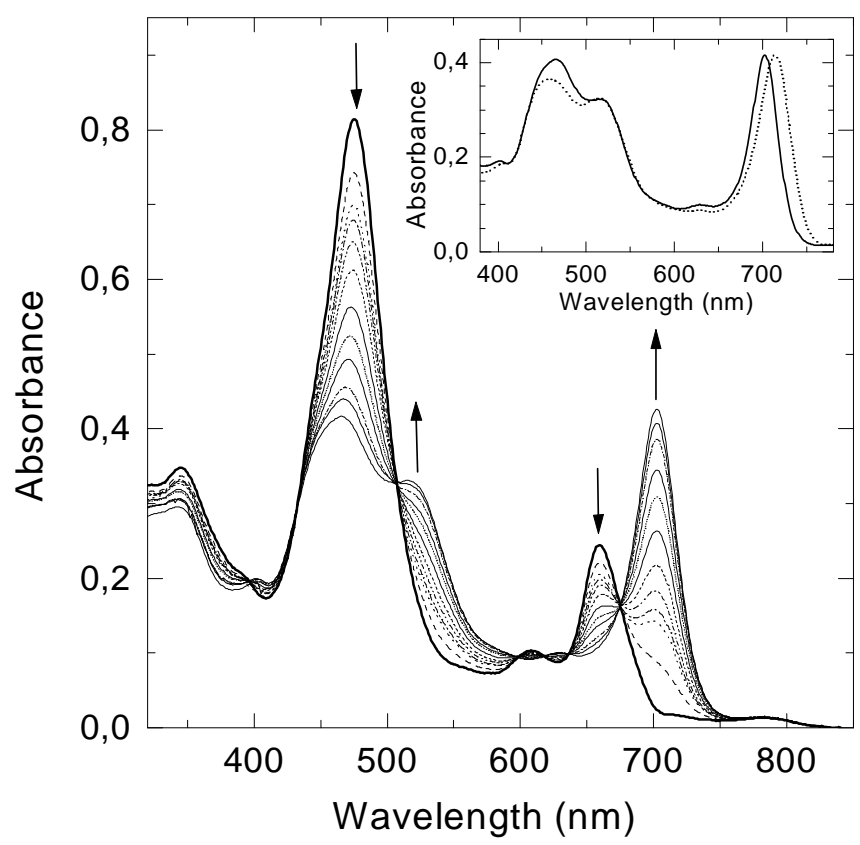


Figure 2

Arellano et al

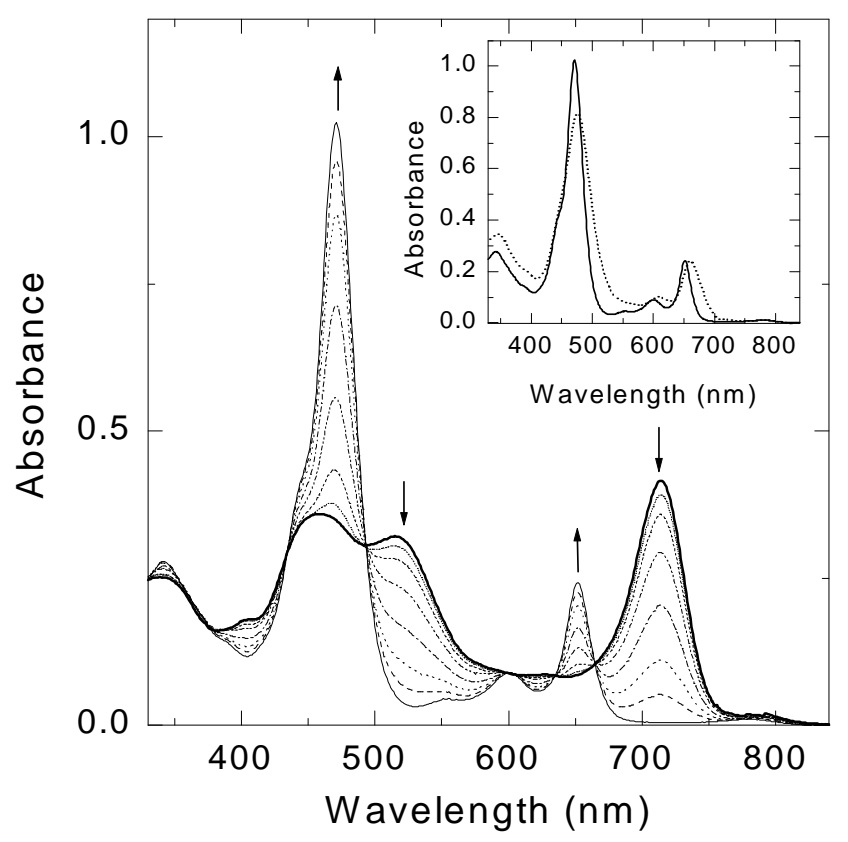


Figure 3

Arellano et al

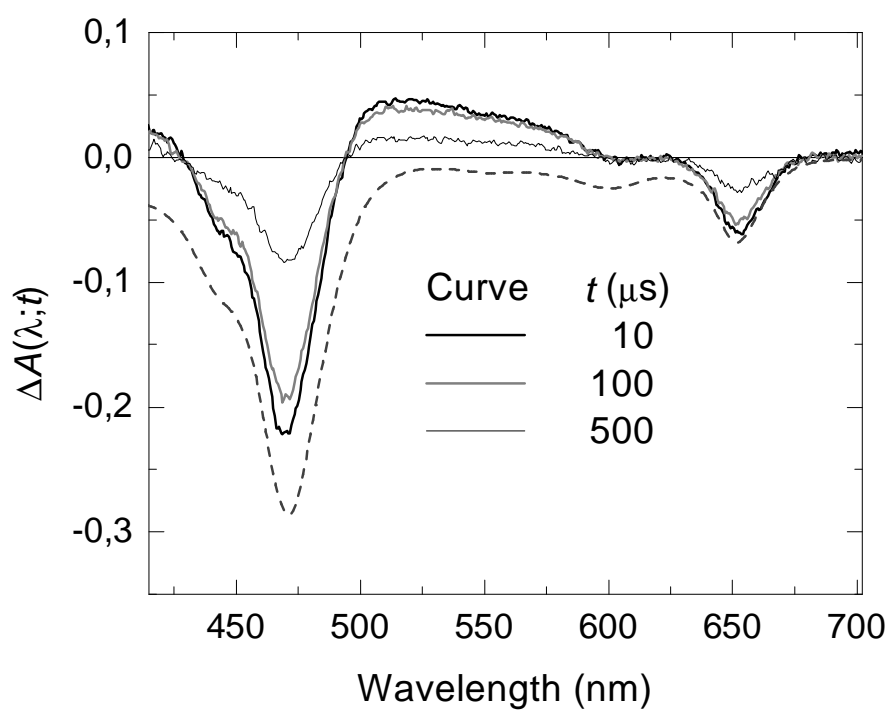


Figure 4

Arellanor et al.

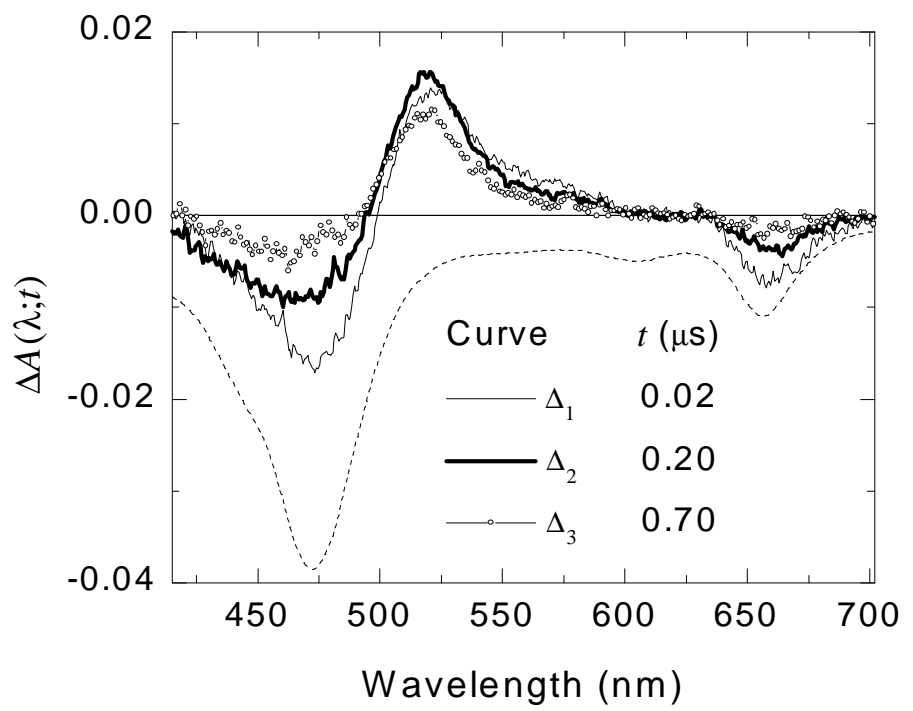


Figure 5

Arellano et al

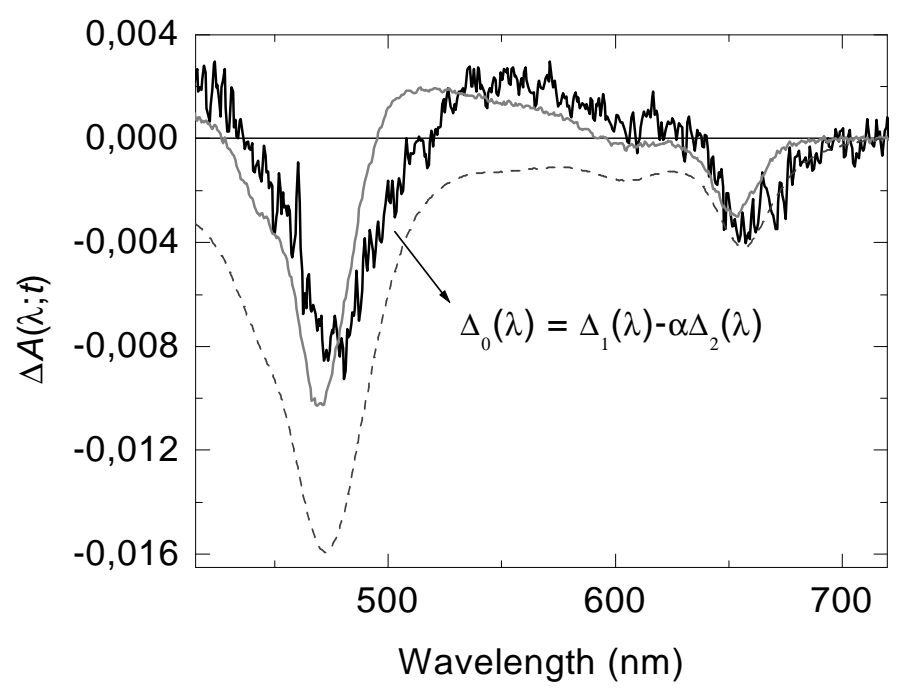


Figure 6

Arellano et al

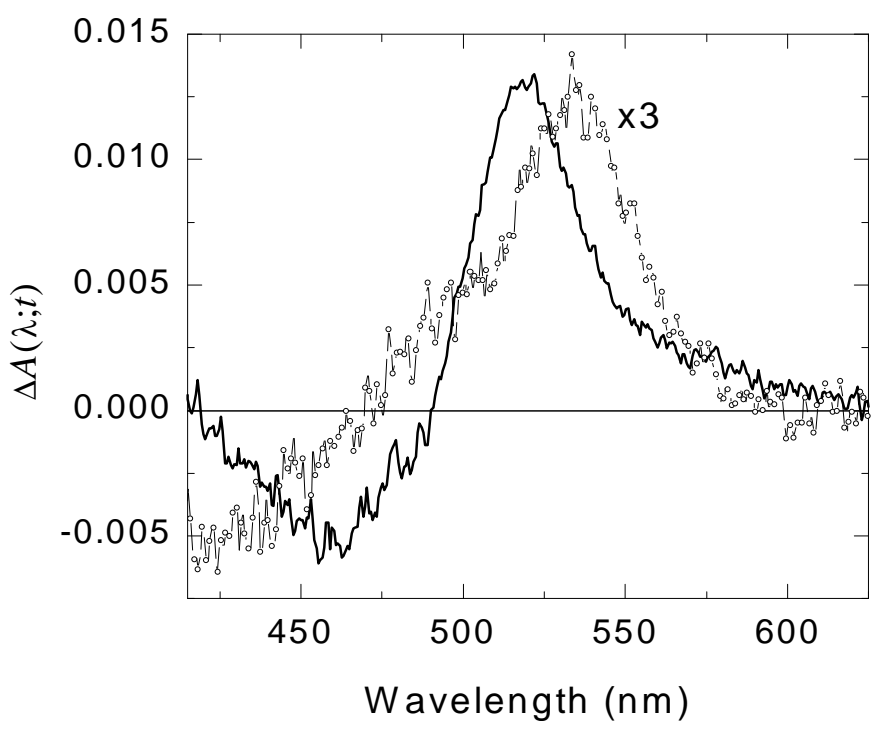


Figure 7

Arellano et al

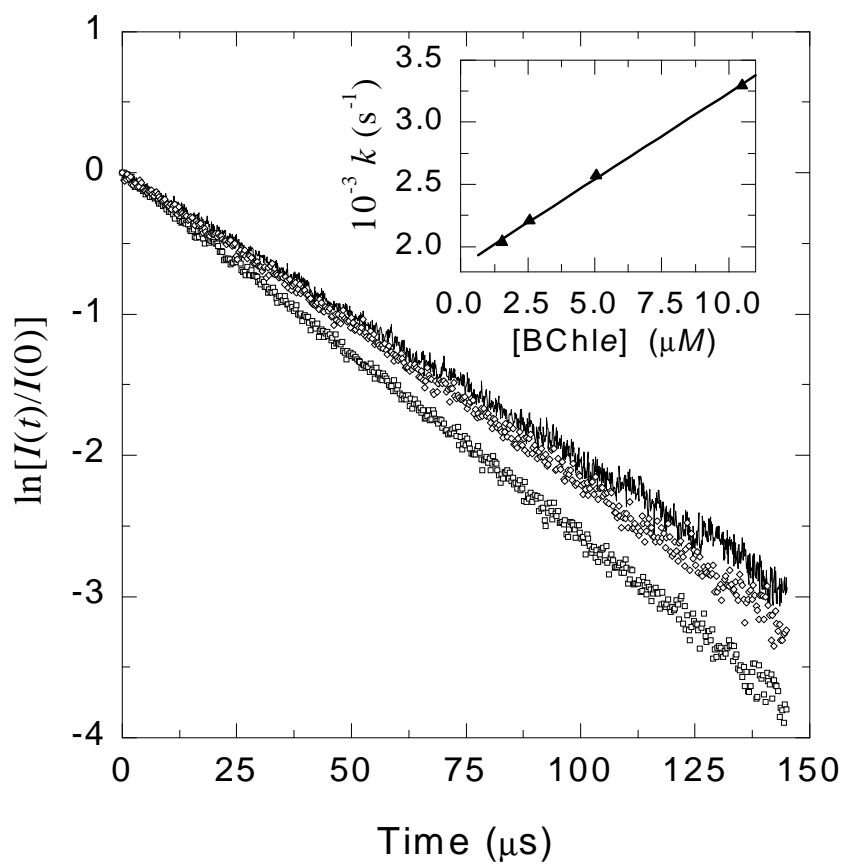


Figure 8

Arellano et al

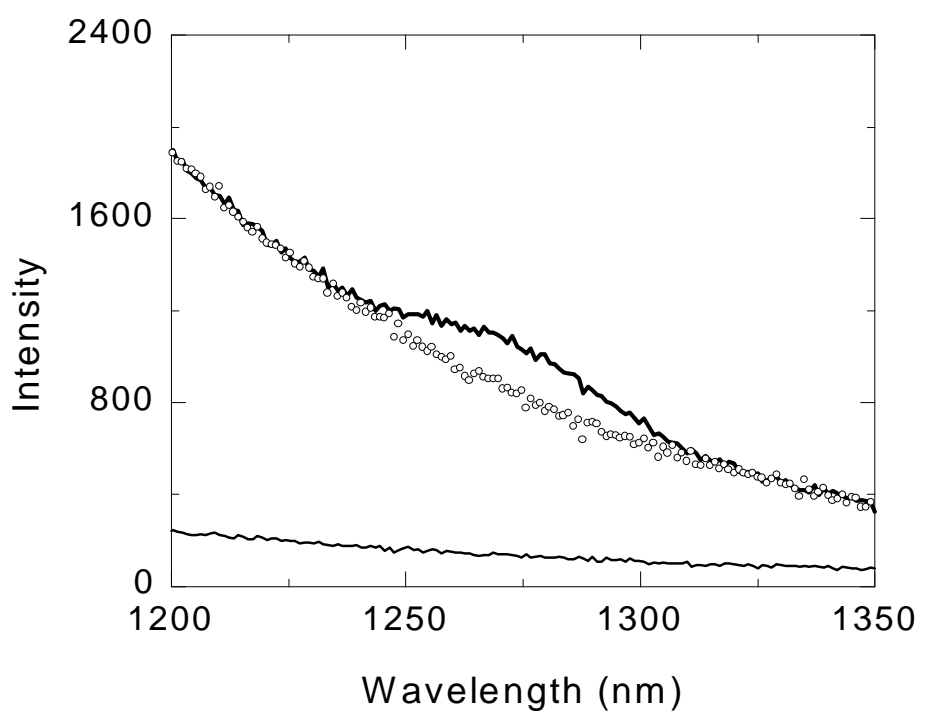

